\title{
SUMMABILITY OF SUBSEQUENCES AND REARRANGEMENTS OF SEQUENCES
}

\author{
THOMAS A. KEAGY
}

\begin{abstract}
Sufficient conditions have been given that require a matrix $A$ to have the property that every sequence $x$ with a finite limit point have a subsequence $y$ such that each finite limit point of $x$ is a limit point of $A y$. In this paper, we show that these conditions may be weakened and obtain an analog in which "subsequence" is replaced with "rearrangement".
\end{abstract}

1. Introduction. The sequence $y$ is a rearrangement of the sequence $x$ if there exists a one-to-one function $\pi$ from the positive integers onto themselves such that for each $k, y_{k}=x_{\pi(k)}$.

The matrix $A$ is a regular summability method if whenever $x$ is convergent, then so is $A x$, and $\lim (A x)_{p}=\lim x_{n}$. The familiar Silverman-Toeplitz characterization of regular matrices consists of the following three conditions.

(1) $\left\{a_{p q}\right\}_{p=1}^{\infty}$ converges to $0, q=1,2,3, \ldots$,

(2) $\left\{\sum_{q} a_{p q}\right\}_{p=1}^{\infty}$ converges to 1 , and

(3) $\sup _{p} \sum_{q}\left|a_{p q}\right|$ is finite.

In [1], R. P. Agnew showed that whenever $x$ is a bounded sequence and $A$ is a regular matrix, then there exists a subsequence $y$ of $x$ such that each limit point of $x$ is a limit point of $A y$. The main purpose of the current paper is to extend this result by weakening the requirement that $A$ be a regular matrix and to obtain an analog to this extension in which "subsequence" is replaced with "rearrangement".

2. Summability of subsequences. D. F. Dawson [4, p. 458] first pointed out the possibility of improving Agnew's result by proving the following theorem.

THEOREM (DAwSON). If $x$ is a complex sequence having a finite limit point and $A$ is a matrix satisfying conditions (1) and (2) of regularity, then there exist a subsequence $y$ of $x$ and an increasing sequence of positive integers $\{p(i)\}_{i=1}^{\infty}$ such that each $(A y)_{p(i)}$ exists and each finite limit point of $x$ is a limit point of $\left\{(A y)_{p(i)}\right\}_{i=1}^{\infty}$.

This theorem strengthens Agnew's result in two ways. The requirement that $A$ be regular is weakened by dropping condition (3) of the Silverman-Toeplitz conditions, and the requirement that $x$ be bounded is replaced with the requirement that $x$ have a finite limit point. But Dawson's theorem does not

Received by the editors April 11, 1977.

AMS (MOS) subject classifications (1970). Primary 40C05, 40A05; Secondary 46B15.

Key words and phrases. Rearrangement, regular summability method. 
imply the existence of $A y$. In the following theorem we complete this generalization in the sense it is shown that the subsequence $y$ may be chosen such that $A y$ does indeed exist.

THEOREM 1. If $x$ is a complex sequence having a finite limit point and $A$ is a matrix satisfying conditons (1) and (2) of regularity, then there exists a subsequence $y$ of $x$ such that each finite limit point of $x$ is a limit point of $A y$.

Proof. Following Agnew [1], we use the separability of the complex plane to form a sequence $u$ with the property that each term of $u$ is a limit point of $x$ and each limit point of $x$ is either one of the terms of $u$ or a limit point of $u$. Let $v=\left\{u_{1} ; u_{1}, u_{2} ; u_{1}, u_{2}, u_{3} ; \ldots\right\}$.

Suppose the increasing sequences of positive integers $\{p(i)\}_{i=1}^{t-1}$ and $\{q(i)\}_{i=1}^{t-1}$ have been defined and the subsequence $\left\{y_{i}\right\}_{i=1}^{q(t-1)-1}$ of $x$ has been determined. Let $p(t)>p(t-1)$ such that

$$
\begin{gathered}
\left|\sum_{q=q(t-1)}^{\infty} a_{p(t), q}-1\right|<1 /\left(2^{t}\left|v_{t}\right|+1\right) \quad \text { and } \\
q(t-1)-1 \\
\sum_{q=1}\left|a_{p(t), q_{q}}\right|<1 / 2^{t} .
\end{gathered}
$$

Let $q(t)>q(t-1)$ such that

$$
\begin{gathered}
\left|\sum_{q=q(t-1)}^{q(t)-1} a_{p(t), q}-1\right|<1 /\left(2^{t}\left|v_{t}\right|+1\right) \text { and } \\
\sup _{p<p(t)}\left|\sum_{q=q(t)}^{k} a_{p q}\right|<1 /\left(2^{t+1}\left|v_{t+1}\right|+1\right)
\end{gathered}
$$

for each $k \geqslant q(t)$. Choose $\left\{y_{i}\right\}_{i=q(t-1)}^{q(t)-1}$ such that $\left\{y_{i}\right\}_{i=1}^{q(t)-1}$ is a subsequence of $x$ and $\left|y_{i}-v_{t}\right|<1 /\left(2^{i+1} \sup _{p}\left|a_{p i}\right|+1\right)$ for $q(t-1)<i<q(t)$. We continue this process so that $\{p(i)\}_{i=1}^{\infty}$ and $\{q(i)\}_{i=1}^{\infty}$ are increasing sequences of positive integers and $y$ is a subsequence of $x$.

We first show that $A y$ is defined. Consider row $p$ of $A$ and let $t=\min \{i$ : $p(i)>p\}$. Then for each $i \geqslant t$,

$$
\begin{aligned}
\left|\sum_{q=q(i)}^{\infty} a_{p q} y_{q}\right| & \leqslant \sum_{j=i}^{\infty}\left|v_{j+1}\right|\left|\sum_{q=q(j)}^{q(j+1)-1} a_{p q}\right|+\sum_{j=i}^{\infty} \sum_{q=q(j)}^{q(j+1)-1}\left|a_{p q}\right|\left|y_{q}-v_{j+1}\right| \\
& <2^{-i}+2^{-q(i)} .
\end{aligned}
$$

But also, if $q(i)<k<q(i+1)$, then

$$
\begin{aligned}
\left|\sum_{q=k}^{q(i+1)-1} a_{p q} y_{q}\right| \leqslant & \left|v_{i+1}\right|\left|\sum_{q=q(i)}^{k-1} a_{p q}\right| \\
& +\left|v_{i+1}\right|\left|\sum_{q=q(i)}^{q(i+1)-1} a_{p q}\right|+\sum_{q=k}^{q(i+1)-1}\left|a_{p q}\right|\left|v_{i+1}-y_{q}\right| \\
& <1 / 2^{i+1}+1 / 2^{i+1}+1 / 2^{k}=1 / 2^{i}+1 / 2^{k}
\end{aligned}
$$


It follows that $(A y)_{p}$ converges for each row $p$; hence $A y$ is defined.

We now show $\lim _{i}\left|(A y)_{p(i)}-v_{i}\right|=0$. This follows since for $i>1$

$$
\begin{aligned}
\left|\sum_{q=1}^{\infty} a_{p(i), q_{q}}-v_{i}\right| \leqslant & \sum_{q=1}^{q(i-1)-1} \mid a_{p(i), y_{q} \mid} \\
& +\left|\sum_{q=q(i-1)}^{q(i)-1} a_{p(i), q_{q}}-v_{i}\right|+\left|\sum_{q=q(i)}^{\infty} a_{p(i), q} y_{q}\right| \\
\leqslant & 1 / 2^{i}+\left|v_{i}\right|\left|\sum_{q=q(i-1)}^{q(i)-1} a_{p(i), q}-1\right|+\sum_{j=1}^{\infty}\left|\sum_{q=q(j)}^{q(j+1)-1} a_{p(i), q}\right|\left|v_{j+1}\right| \\
& +\sum_{j=i-1}^{\infty} \sum_{q=q(j)}^{\infty+1)-1}\left|a_{p(i), q}\right|\left|y_{q}-v_{j+1}\right| \\
& <1 / 2^{i}+1 / 2^{i}+1 / 2^{i}+1 / 2^{q(i-1)} .
\end{aligned}
$$

3. Summability of rearrangements. J. A. Fridy [5, p. 189] has obtained an analog to Agnew's result in which he replaces "subsequence" with "rearrangement". It is natural to ask whether Fridy's result may be extended in the same sense that Theorem 1 extended Agnew's result. The answer to this question is "yes", and the following theorem will prove useful in establishing such an analog to Theorem 1 involving rearrangements.

THEOREM 2. Let $A$ be a matrix with null rows and columns and $x$ be $a$ complex sequence. If $y$ is a subsequence of $x$ such that Ay exists and has a finite limit point, then there exists a rearrangement $z$ of $x$ such that Az exists and each finite limit point of $A y$ is a limit point of $A z$.

Proof. As in the proof of Theorem 1, we construct a sequence $u$ such that each $u_{i}$ is a limit point of $A y$ and each limit point of $A y$ is a term of $u$ or a limit point of $u$. Let $v=\left\{u_{1} ; u_{1}, u_{2} ; u_{1}, u_{2}, u_{3} ; \ldots\right\}$.

Suppose that the increasing sequences of positive integers $\{p(i)\}_{i=1}^{t-1}$ and $\{q(i)\}_{i=1}^{t-1}$ have been chosen and that $\left\{z_{i}\right\}_{i=1}^{q(t-1)}$ is a permutation of $q(t-1)$ terms of $x$. Let $k=\min \left\{j: x_{j} \notin\left\{z_{i}\right\}_{i=1}^{q(t-1)} \cup\left\{y_{i}\right\}_{i=q(t-1)+1}^{\infty}\right\}$. Let $p(t)>p(t$ $-1)$ such that $\sum_{q=1}^{q(t-1)}\left|a_{p(t), q} z_{q}\right|<1 / 2^{t}$ and

$$
\left|\sum_{q=q(t-1)+1}^{\infty} a_{p(t), q_{q}}-v_{t}\right|<1 / 2^{t} .
$$

Let $q(t)>q(t-1)$ such that

$$
\begin{gathered}
\left|\sum_{q=q(t-1)+1}^{q(t)-1} a_{p(t), y_{q}}-v_{t}\right|<1 / 2^{t}, \\
\left|\sum_{q=q(t)}^{\infty} a_{p(t), y_{q}} y_{q}\right|<1 / 2^{t} \text { and } \sup _{p<p(t)}\left|x_{k}-y_{q(t)}\right|\left|a_{p, q(t)}\right|<1 / 2^{t+1} .
\end{gathered}
$$


Let $z_{q}=y_{q}$ for $q(t-1)<q<q(t)$ and $z_{q(t)}=x_{k}$. We continue this process so that $\{p(i)\}_{i=1}^{\infty}$ and $\{q(i)\}_{i=1}^{\infty}$ are increasing sequences of positive integers and $z$ is a rearrangement of $x$.

We first show that $A z$ is defined. Consider row $p$ of $A$ and let $t=\min \{i$ : $p(i)>p\}$. Let $k>q(t)$ and $j=\max \{i: k \geqslant q(i)\}$. Then

$$
\left|\sum_{q=k}^{\infty} a_{p q} z_{q}\right| \leqslant\left|\sum_{q=k}^{\infty} a_{p q} y_{q}\right|+\sum_{i=j}^{\infty}\left|y_{q(i)}-z_{q(i)}\right|\left|a_{p, q(i)}\right|<\left|\sum_{q=k}^{\infty} a_{p q} y_{q}\right|+1 / 2^{j},
$$

and it follows that $(A z)_{p}$ converges.

Next, we show that $\left|(A z)_{p(t)}-v_{t}\right|<1 / 2^{t-2}$. This inequality holds since

$$
\begin{aligned}
\left|\sum_{q=1}^{\infty} a_{p(t), q} z_{q}-v_{t}\right| \leqslant & \sum_{q=1}^{q(t-1)}\left|a_{p(t), q_{q}}\right|+\left|\sum_{q=q(t-1)+1}^{q(t)-1} a_{p(t), q_{q}}-v_{t}\right| \\
& +\sum_{j=t}^{\infty}\left|y_{q(j)}-z_{q(j)}\right|\left|a_{p(t), q(j)}\right|+\left|\sum_{q=q(t)}^{\infty} a_{p(t), q_{q}}\right| \\
& <1 / 2^{t}+1 / 2^{t}+1 / 2^{t}+1 / 2^{t} .
\end{aligned}
$$

Hence each limit point of $A y$ is a limit point of $A z$, and the proof is complete.

The form of Theorem 2 was chosen in order to simplify the details of the proof. Actually, a slightly more general result may be shown by using the basic structure of the above proof. For instance, it is not necessary to require that each column of $A$ be null, an infinite number of null columns will suffice. Also, in order to show that $A z$ exist, it is not necessary to require that Ay have a finite limit point. The theorem in its present form does, however, provide a sufficient tool to enable us to establish the following analog to Theorem 1.

THEOREM 3. If $x$ is a complex sequence having a finite limit point and $A$ is a matrix satisfying properties (1) and (2) of regularity, then there exists a rearrangement $y$ of $x$ such that each finite limit point of $x$ is a limit point of $A y$.

The proof of Theorem 3 is an immediate consequence of Theorems 1 and 2 and will be omitted.

Corollary 4. A complex sequence $x$ diverges to $\infty$ if there exists a matrix $A$ satisfying conditions (1) and (2) of regularity such that Ay diverges to $\infty$ for every rearrangement $y$ of $x$.

The proof of Corollary 4 parallels the argument given by Dawson [4, p. 459] for a similar result involving subsequences and will be omitted.

THEOREM 5. If $x$ is a complex sequence and $A$ is a row-finite matrix satisfying conditions (1) and (2) of regularity, then there exists a rearrangement $y$ of $x$ such that every limit point of $x$ (finite or infinite) is a limit point of Ay. 
Proof. We assume without loss of generality that $x$ is unbounded and has at least one finite limit point. Let $v$ be as defined in the proof of Theorem 1 . Suppose the increasing sequences of positive integers $\{p(i)\}_{i=1}^{t-1},\{r(i)\}_{i=1}^{t-1}$, $\{k(i)\}_{i=1}^{t-1}$, and $\{q(i)\}_{i=1}^{t-1}$ have been selected and that $\left\{z_{i}\right\}_{i=1}^{q(t-1)}$ is a permutation of $q(t-1)$ terms of $x$. Let $p(t)>r(t-1)$ such that $\sum_{q=1}^{q(t-1)}\left|a_{p(t), q} z_{q}\right|<$ $1 / 2^{t}$ and

$$
\left|\sum_{q=q(t-1)+1}^{\infty} a_{p(t), q}-1\right|<1 / 2^{t}\left(\left|v_{t}\right|+1\right) .
$$

Let $k(t)=\sup \left\{q:\left|a_{p(t), q}\right|>0\right\}$ and choose $\left\{z_{i}\right\}_{i=q(t-1)+1}^{k(t)}$ from $x \backslash\left\{z_{i}\right\}_{i=1}^{q(t-1)}$ such that $\left|\sum_{q=q(t-1)+1}^{k(t)} a_{p(t), q} z_{q}-v_{t}\right|<1 / 2^{t}$. Let $z_{k(t)+1}=x_{w}$ where $w=$ $\min \left\{j: x_{i} \notin\left\{z_{i}\right\}_{i=1}^{k}\right\}$. Since $A$ has properties (1) and (2) of regularity, there exist $r(t)>p(t)$ and $q(t)>k(t)+1$ such that $\left|a_{r(t), q(t)}\right|>0$ and $\left|a_{r(t), q}\right|=0$ whenever $q>q(t)$. Choose $\left\{z_{i}\right\}_{i=k(t)+2}^{q(t)-1}$ from $x \backslash\left\{z_{i}\right\}_{i=1}^{k(t)+1}$ and let $z_{q(t)} \in$ $x \backslash\left\{z_{i}\right\}_{i=1}^{q(t)-1}$ such that $\left|\sum_{q=1}^{q(t)} a_{r(t), q} z_{q}\right|>2^{t}$. This selection process may be repeated yielding the increasing sequences of positive integers $\{p(i)\}_{i=1}^{\infty}$ and $\{r(i)\}_{i=1}^{\infty}$ and the rearrangement $z$ of $x$ having the property that $\left|(A z)_{p(t)}-v_{t}\right|$ $<1 / 2^{t-1}$ and $\left|(A z)_{r(t)}\right|>2^{t}$ for each $t$.

COROLlary 6. $A$ complex sequence $x$ is bounded if there exists a matrix $A$ satisfying conditions (1) and (2) of regularity such that $A y$ is bounded for every rearrangement $y$ of $x$.

The proof of Corollary 6 parallels the argument given by Dawson [4, p. 459] for a similar result involving subsequences and will be omitted.

\section{REFERENCES}

1. R. P. Agnew, Summability of subsequences, Bull. Amer. Math. Soc. 50 (1944), 596-598. MR 6, 46.

2. R. C. Buck, A note on subsequences, Bull. Amer. Math. Soc. 49 (1943), 898-899. MR 5, 117.

3. 1074-1075. MR 18, 478.

4. D. F. Dawson, Summability of subsequences and stretchings of sequences, Pacific J. Math. 44 (1973), 455-460. MR $47 \$ 5478$.

5. J. A. Fridy, Summability of rearrangements of sequences, Math. Z. 143 (1975), 187-192. MR $52 \$ 3772$.

6. I. J. Maddox, A Tauberian theorem for subsequences, Bull. London Math. Soc. 2 (1970), 63-65. MR $41 \sharp 5836$.

7. T.A. Keagy, Summability of certain category two classes, Houston J. Math. 3 (1977), 61-65.

Department of Mathematics, TeXas Eastern University, Tyler, TeXas 75701 